\section{Vertebrate ovary}

The Vertebrate Ovary: Comparative Biology and Evolution. Edited by R. E. Jones. Pp. 853. (Plenum: New York and London, 1978.) $£ 43.78$.

Publication of another volume (over 800 pages) on the ovary highlights the expansion of research in this aspect of reproductive biology. Consisting of 24 reviews by leading scientists predominantly from the USA the work is mostly comparative in nature. This is exemplified at the beginning and end of the book by chapters on the origin and segregation of primordial germ cells, in which the anuran germ line receives special attention, and on the evolution of the vertebrate ovary. Morphological differentiation of the ovary is also considered from a comparative viewpoint, but biochemical and genetic factors that influence gonadal differentiation are treated only briefly.

The follicle is reviewed extensively: its genesis, morphology and endocrine function; the hormonal control of its growth and maturation; and the production of follicular fluid. The nonatretic follicle is seen as developing in a predominantly oestrogenic microenvironment that influences follicular growth; processes that result in atresia, and the repercussion of this phenomenon on ovarian function, are examined separately.

Understandably, formation of oogonia, maturation of the oocyte and ovulation receive much attention. Most contributors dealing with mechanistic aspects of ovarian physiology summarise their ideas in the form of hypothesis. This is seen in the description of ovulation, and of oocyte maturation in amphibians, in which the elaborate biochemical events induced by steroids that lead to the formation of the meiotic spindle are examined. Work on this latter aspect contrasts with experiments in mammals which have focused rather on the maturationinducing action of luteinising hormone and on how the resumption of meiosis is prevented by granulosa cells and follicular fluid inhibitor.

Compared with its close contemporary, The Ovary (edited by Lord Zuckerman and B. J. Weir; second edition; Academic: London and New York; three volumes; total price $£ 77.70$ ), it is inevitable that this new book contains chapters on similar topics. However, interpretations by different experts frequently result in essays that are complementary rather than merely repetitious. Valuable additions include reviews of ovarian vasculature, with a description of techniques of blood flow measurement and of the effect of humoral agents, ovarian innervation, and the fascinating fields of follicular selection and vertebrate fecundity. Discussion of the fate of the ruptured follicle is less satisfactory, as reference to the evolutionary significance of the corpus luteum and the comparative aspects of its structure and function are found dispersed among several reviews. The lack of chapters on ovarian pathology and the influence of external factors on ovarian function is attributed to the restrictions of space, but for a book of this quality the reproduction of photomicrographs deserves a better standard.

A selected sentence from a paper by Bern (1972) reffects the editor's bias: "we comparative biologists have an aim-the aim to reconstruct from extant species a picture of the evolution of the system in which we are interested". The picture proves complex, frequently displaying diversity rather than conformity. Nonetheless the book should prove valuable for the research scientist, clinician and teacher; and the nature of unanswered questions should stimulate enquiry.

R. B. Heap

R. B. Heap is Head of the Department of Physiology at the $A R C$ Institute of Animal Physiology, Babraham, Cambridge, UK.

\section{Climatic impact on organic evolution}

Climate and Evolution. By Ronald Pearson. Pp. 274. (Academic: London, New York and San Francisco, 1979.) $£ 14$.

THIS is an ambitious work that must be taken seriously by anyone interested in palaeoclimatology and biostratigraphy. Such a study, in order to fulfil its title, requires a multi-disciplinary approach to a vast literature and is more than an ordinary mortal could achieve. Pearson, however, is mortal enough, so it is worth seeing where he succeeds and where the book falls short.

The book is organised so that chapters 1-5 are general and 6-11 historical. The introduction concerns evolution only and is brief but to the point. Chapters 2, "A brief history of historical climatology", and 3, "More recent knowledge of climatic change", review the principal hypotheses for climatic change but primarily for changes evident in Quaternary history. This is to some extent balanced by chapter 5, "Long term considerations", with chapter 4, "Geomagnetic considerations", thrown in for good measure. The result is a very brief resume of the mechanisms for climatic change abstracted from many leading authorities in these fields. The remaining chapters are chronological: (6) Palaeozoic; (7) Mesozoic; (8) Tertiary; (9) Quaternary; (10) Late Weichselian and Flandrian; and (11) Climate and [human] history. A consolidated list of more than 750 references and a fairly good index, complete the book.

The author's own viewpoint is evident in the balance of the work. His own publications listed concern Quaternary Coleoptera; and the whole discus- sion is Quaternary-orientated, so that earlier and possibly more radical climatic and evolutionary changes are increasingly obscured through the mists of time. Precambrian history is accorded token mention and the first metazoan (Ediacaran) fauna (surely of prime significance in such a study) receives only a passing reference without definite mention. In the same chapter discussion of climatic change on the megascale is minimal. For example, the role of atmospheric $\mathrm{CO}_{2}$ is hardly discussed and the possibility of the first half of the Earth's history being climatically hotter than later because of this and other factors, is not considered. Inevitably too, the consideration of Pre-Quaternary history depends on more stratigraphical knowledge than is easy to acquire for the purposes of such a work.

An alternative approach might have been more satisfactory, namely, to review organic evolution and first identify the developments that need explanation and then to compare climatic and other mechanisms in each case against a critical assessment of the chronolobear. And here is a difficulty: inevitably the many chronometric age estimates quoted tend to be taken at their numerical face value, and it would require much research to do otherwise.

In conclusion, this work, except for parts of the Quaternary record, does not provide a critical assessment of the climatic impact on organic evolution but it does a most valuable service in bringing together and abstracting a variety of relevant literature and the wide range of ideas necessary as a preliminary. Most specialists will find something here to challenge their thinking.

\section{W. B. Harland}

W. B. Harland is Reader in Tectonic Geology at the University of Cambridge, UK. gical precision of the data brought to 